\title{
A New Proportional-Share Disk Scheduling Algorithm: Trading-Off I/O Throughput and QoS Guarantee
}

\author{
Young Jin Nam and Chanik Park \\ Department of Computer Science and Engineering \\ Pohang University of Science and Technology/PIRL \\ Kyungbuk, Republic of Korea \\ $\{$ y jnam, cipark\}@postech.ac.kr
}

\begin{abstract}
Proportional-share algorithms are designed to allocate an available resource, such as a network, processor, or disk, for a set of competing applications in proportion to the resource weight allotted to each. While a myriad of proportional-share algorithms were made for network and processor resources, little research work has been conducted on disk resources which exhibit non-linear performance characteristics attributed to disk head movements. This paper proposes a new proportional-share disk scheduling algorithm which accounts for overhead caused by disk head movements and QoS guarantees in an integrated manner. Performance evaluations via simulations reveal that the proposed algorithm improves I/O throughput by $11-19 \%$ with only $1-2 \%$ QoS deterioration.
\end{abstract}

\section{Introduction}

The prevalence of streaming services increases the odds of sharing disk resources. As a result, the traffic control on the disk resource called storage QoS (Quality of Service) has been gaining in significance in order to satisfy the requirements of different applications. It is known that partitioning such disk resources as bandwidth plays a key role in satisfying the different QoS requirements of various types of applications, such as best-effort applications and real-time applications [1]. Despite its importance, research on storage QoS is still in its infancy, having mainly focused on an underlying disk scheduling algorithm.

A disk resource exhibits different characteristics from other resource types, such as processors and networks because high overhead is typically involved in processing I/O requests. Few disk scheduling algorithms proportionally share a disk resource, such as YFQ [2] and Cello [1]. Among them, the YFQ algorithm is based on packet-based fair queuing algorithms in the network, i.e., WFQ [3] to choose a subsequent I/O request to be scheduled and SFQ [4] to maintain

\footnotetext{
* The authors would like to thank the Ministry of Education of Korea for its financial support toward the Electrical and Computer Engineering Division at POSTECH through its BK21 program. This research was also supported in part by HY-SDR IT Research Center.
} 
a global virtual time. Considering that achieving the ultimate QoS guarantee requires integrated scheduling and management for various resources in an underlying system, such as a processor, network, or disk, the YFQ-like approach employing a packet-based fair queuing algorithm is more desirable. Unfortunately, proportional-share disk scheduling algorithms, while preserving a given QoS feature, inevitably suffer from performance degradation. In order to improve their disk I/O performance, the YFQ algorithm attempts to select I/O requests mainly based on a QoS guarantee, and then attempts to reduce disk overhead by batching to the set of $\mathrm{I} / \mathrm{O}$ requests selected in the previous phase. After investigating the operations of this scheduling algorithm, we determined that the effectiveness of disk overhead reduction is restricted by separating the operation of $\mathrm{I} / \mathrm{O}$ request selection from the operation of reducing disk overheads. Moreover, while we can achieve a better I/O performance with a larger batch size, the size of the batch cannot be arbitrarily increased in actual systems.

This paper proposes a new proportional-share disk scheduling algorithm which considers the issues of disk I/O overhead reduction and I/O bandwidth provisioning in an integrated manner, thus overcoming the shortcoming of limited batch sizes found in the YFQ algorithm. Our basic idea derives from the fact that combining the operation of $\mathrm{I} / \mathrm{O}$ request selection with the operation of reducing disk overheads will increase the odds of reducing disk overheads while maintaining a certain level of QoS guarantee.

\section{The Proposed Algorithm}

We begin by providing a few notations. Assume that $N$ different I/O workloads exist. The $k$-th reservation queue for the $k$-th $\mathrm{I} / \mathrm{O}$ workload is denoted as $R Q_{k}=\left\{r_{k}^{1}, r_{k}^{2}, \ldots\right\}$, where $r_{k}^{i}$ refers to the $i$-th I/O request in the $k$-th reservation queue. The notation of $l_{k}^{i}$ represents the request size of $r_{k}^{i}$. $R Q_{k}$ requires a different QoS requirement denoted with $w_{k}$; that is, a different amount of disk bandwidth. Actual bandwidth alloted to the $k$-th $\mathrm{I} / \mathrm{O}$ workload is $\sum_{i} w_{k} w_{i} B$, where $B$ is the disk bandwidth. As with packet-based fair queuing algorithms [3, 4], each reservation queue $R Q_{k}$ maintains a virtual start time and a virtual finish time, denoted by $S_{k}$ and $F_{k}$, respectively. Besides, a global virtual time $v(t)$ is maintained.

\subsection{Overall Architecture}

The proposed algorithm consists of a set of reservation queues $(R Q)$, the base QoS sequence generation (BQS) module, and the disk overhead reduction (DOR) module, as shown in Figure 1. The reservation queues are used by the BQS and DOR modules. The proposed algorithm aims to produce an expanded sequence of I/O requests denoted with $S_{\text {expanded }}$, which not only preserves a given QoS feature, but also achieves a better disk I/O performance based on a base sequence of I/O requests denoted with $S_{\text {base }}$, which is generated by the strict QoS-enforcing 


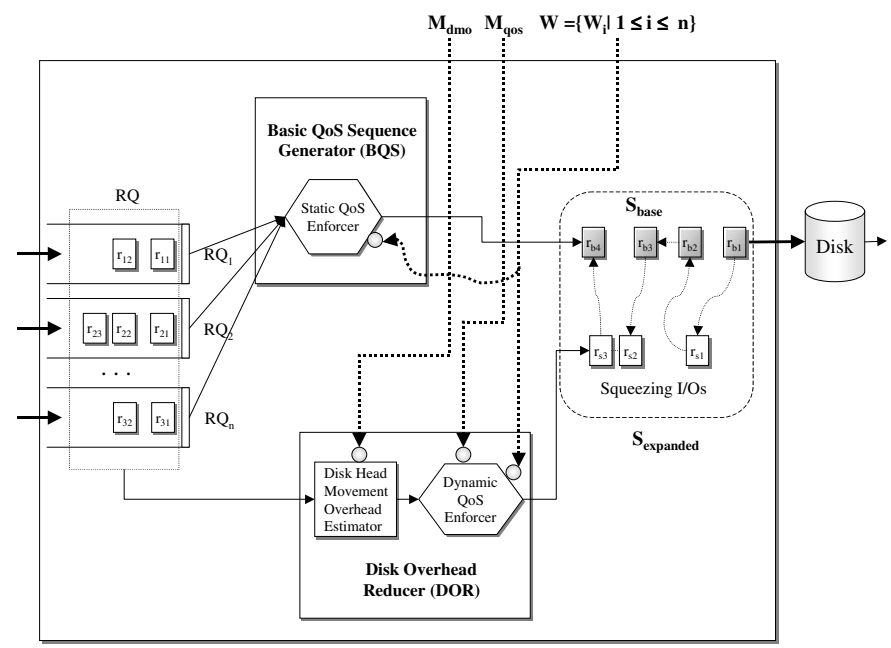

Fig. 1. Architecture of the proposed algorithm

module (the BQS module). Each I/O workload has its own reservation queue called $R Q_{k}$ to which a corresponding disk bandwidth $w_{k}$ is allocated.

The BQS module generates a $S_{\text {base }}$ from I/O requests in $R Q$ as with the YFQ algorithm. First, it selects an I/O request from the reservation queue of the minimum virtual finish time, say $r_{s}^{1}$ and $R Q_{s}$, respectively. Second, it advances $v(t)=S_{s}$, Third, it updates $S_{s}=F_{s}$ and $F_{s}=S_{s}+\frac{l_{s}^{\text {next }}}{w_{s}}$ if $R Q_{s}$ has a next $\mathrm{I} / \mathrm{O}$ request, where $l_{s}^{\text {next }}$ is the size of the next I/O request of $r_{s}^{1}$. Otherwise, $S_{s}$ and $F_{s}$ remain unchanged. If a new request $r_{i}^{k}$ arrives at the empty $R Q_{i}$, then $S_{i}=\max \left\{v(t), F_{i}\right\}$ and $F_{i}=S_{i}+\frac{l_{i}^{k}}{w_{i}}$. The typical number of I/O requests selected by the BQS module is four. After choosing a number of I/O requests, the BQS module reorders them in a $\mathrm{C}-\mathrm{SCAN}$ order, thus producing a final sequence of I/O requests called a base I/O sequence, $S_{\text {base }}$.

The DOR module generates $S_{\text {expanded }}$ by attempting to insert as many I/O requests as possible into $S_{\text {base }}$ given by the BQS module when two conditions on the disk idle time and QoS guarantee are met. These two conditions are determined by two controlling parameters, $M_{d m o}$ and $M_{q o s}$. (These two parameters will be explained in the succeeding paragraph.) The DOR module consists of two core components: the Disk Head Movement Overhead Time Estimator (DME) and the Dynamic QoS Enforcer (DQE). The DME module determines whether an extra I/O request in $R Q$ can be squeezed into a $S_{\text {interim }}$ by estimating the disk overhead time which exists in the sequence. $S_{\text {interim }}$ means an intermediate I/O sequence in transit from $S_{\text {base }}$ to $S_{\text {expanded }}$. To control the insertion of I/O requests into the current $\mathrm{I} / \mathrm{O}$ sequence, we devise a controlling parameter called an overhead margin $M_{d m o}$, which will eventually be added to the estimated disk overhead time between the two I/O requests in $S_{\text {interim }}$. As $M_{d m o}$ becomes larger, a chance increases that an extra $\mathrm{I} / \mathrm{O}$ request can be squeezed into the 
sequence. The DQE module controls the degree of QoS enforcement against the I/O requests which have passed the DME module successfully. Aside from the start/finish virtual times at each queue, this module additionally maintains a separate set of virtual times for each $R Q_{k}$ and a displacement between the maximum and minimum virtual times among the non-empty reservation queues. A virtual time can advance as long as the displacement is not greater than the QoS margin denoted with $M_{\text {qos }}$. We will define two groups of I/O requests: the BQS_IO and the DOR_IO. The BQS_IO represents a group of I/O requests which pass through the BQS module, and the DOR_IO means a group of I/O requests squeezed into a base I/O sequence $S_{\text {base }}$ via the DOR module. Obviously, all I/O requests of the BQS_IO can commit a given QoS guarantee.

\section{$2.2 M_{d m o}, M_{q o s}$ Control and Algorithm Description}

We will explore in detail the behavior of the DME and DQE modules with the two controlling parameters: $M_{d m o}$ and $M_{q o s}$. $M_{d m o}$ determines a margin for a disk overhead time which exists between two I/O requests in $S_{\text {interim }}$, and $M_{q o s}$ decides how rigorously a given $\mathrm{QoS}$ feature is to be applied to the $\mathrm{I} / \mathrm{O}$ requests which have been chosen by the DME module. The size and I/O characteristics of a $S_{\text {expanded }}$ depend heavily on the $M_{d m o}$ and $M_{\text {qos }}$ values.

To take control of inserting I/O requests to the current I/O sequence, we introduce $M_{d m o}$ to be added to the estimated disk overhead time between the two I/O requests in $S_{\text {interim. }}$. Property 1 clarifies the meaning of the marginal overhead time $M_{d m o} . T_{o v}\left(r_{i}, r_{j}\right)$ means the disk overhead time from request $r_{i}$ to $r_{j}$ and the disk $S_{\text {interim }}$ represents an I/O sequence in transit from $S_{\text {base }}$ to $S_{\text {expanded. }}$.

Property 1. $r_{k}$ can be insertable between $r_{i}$ and $r_{j}$ in $S_{\text {interim }}$, if $T_{o v}\left(r_{i}, r_{k}\right)+$ $T_{o v}\left(r_{k}, r_{j}\right) \leq T_{o v}\left(r_{i}, r_{j}\right)+M_{d m o}$.

The $M_{d m o}=p$ implies that its overhead margin is equal to the $p$ percentage of the full seek time of an underlying disk. Even though the disk overhead between two I/O requests can be defined in several ways, we simply use a seek overhead between $r_{i}$ and $r_{j}$. The information on the seek times of an underlying disk can be extracted from host [5]. According to Property 1, we expect that a length of $S_{\text {expanded }}$ will be longer in proportion to the value of $M_{d m o}$. However, recall that a length of $S_{\text {expanded }}$ is also affected by $M_{\text {qos }}$.

Next, we maintain another QoS enforcement scheme that consists of a set of virtual times for each $R Q_{k}$ and a current virtual time displacement, in order to enforce a given QoS feature on I/O requests which have been successfully passed by the DME module. The purpose of enforcing a given QoS is to keep a given displacement controlled by the $M_{\text {qos }}$ parameter for the virtual times of the non-empty reservation queues. Denote the additional virtual time of $R Q_{k}$ with $V_{k}$. Initially, $V_{k}=0$. If an I/O request arrives at an empty $R Q_{k}$, $V_{k}=\max \left\{V_{k}, \min _{\{j, j \neq k\}}\left\{V_{j}\right\}\right\}$, for $R Q_{j} \neq \emptyset$. The displacement among different virtual times is written as $\delta_{V}=\max _{i}\left\{V_{i}\right\}-\min _{j}\left\{V_{j}\right\}$ for the non-empty reservation queues. 
Property 2. For a $r_{k}^{i} \in R Q_{k}, r_{k}^{i}$ can preserve a given QoS feature if it meets: $\delta_{V} \leq M_{\text {qos }}$ when $V_{k}=V_{k}+\frac{l_{k}^{i}}{w_{k}}$.

$M_{q o s}=q$ implies that a set of virtual times will advance with the following amount of QoS deterioration, such that the resulting I/O throughput of each $R Q_{k}$ will deviate from a given $\mathrm{QoS}$ feature on the average by $\frac{w_{k}}{l_{k}^{a g} g} \mathrm{I} / \mathrm{O}$ requests, where $l_{k}^{a v g}$ is an average block size of an $\mathrm{I} / \mathrm{O}$ request from $R Q_{k}$. According to Property 2, no I/O requests can pass the DOR module with $M_{q o s}=0$, since no displacement is allowed. It implies that a given QoS feature is preserved strictly with the BQS module.

Algorithm Description: Algorithm 1 describes how the DOR module operates. Recall that the BQS module strictly enforces a given QoS feature in exactly the same manner as the YFQ algorithm.

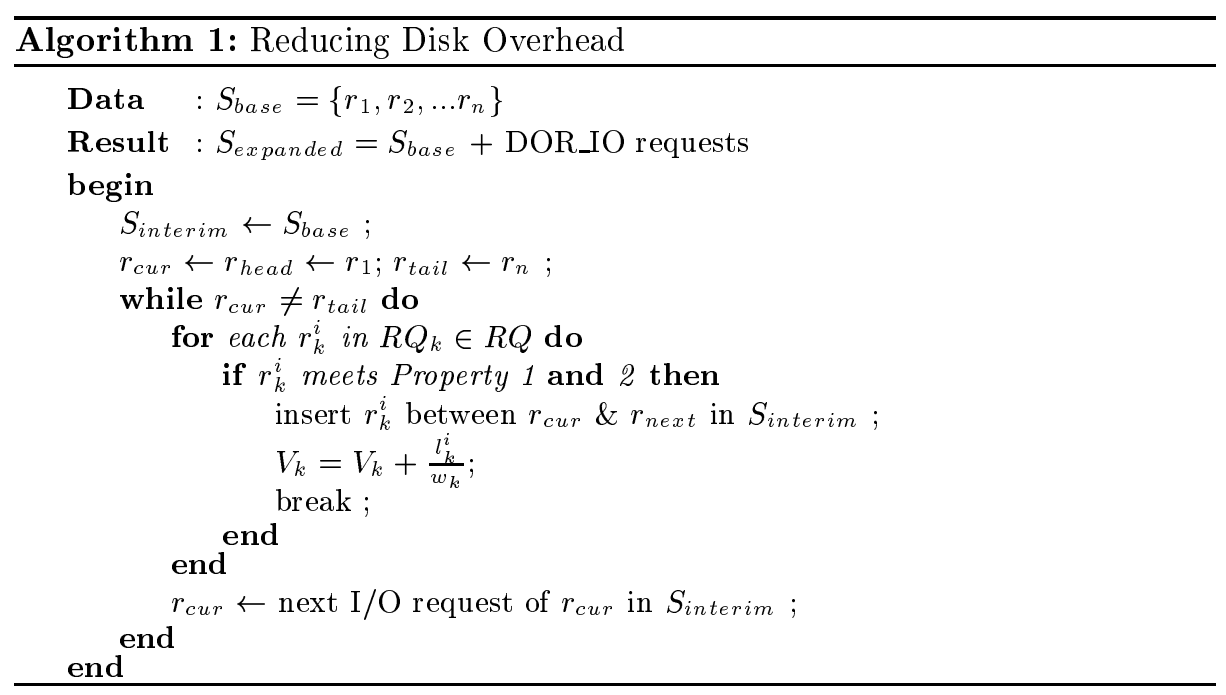

\section{Performance Evaluations}

This section investigates the performance of the proposed algorithm by discovering two desirable values of $M_{d m o}$ and $M_{q o s}$ which not only improve disk I/O performance, but also preserve a given QoS feature with negligible deterioration.

\subsection{Simulation Environments}

We implemented the proposed algorithm as one of driver-specific disk scheduling schedulers within the DiskSim simulator [6]. Two competing I/O workloads are 
synthetically generated. The sizes of the I/O requests are distributed normally with a mean of 8 blocks. The ratio of reads to writes is set to two. An I/O workload level becomes heavier by increasing the number of outstanding I/O requests denoted with $|I O|$. A start block address of each I/O request is distributed randomly over the entire area of a IBM DNES 309170W SCSI disk which serves arriving $\mathrm{I} / \mathrm{O}$ requests in a FIFO manner with a maximum of four outstanding concurrent I/O requests. It is assumed that two reservation queues exist, which are $R Q_{1}$ and $R Q_{2} \cdot w_{1}=80$ and $w_{2}=20$. With the QoS requirements, the BQS module selects four I/O requests from the head of the reservation queues and then generates $S_{\text {base }}$ where the I/O requests are reordered in a C-SCAN order. Next, the DOR module generates $S_{\text {expanded }}$ by expanding a given $S_{\text {base }}$ with a group of squeezing I/O requests. As noted, two controlling parameters exist, which are an overhead margin of $M_{d m o}$ for the DME module and a QoS margin of $M_{q o s}$ for the DQE module. A combination of these two parameters determines the length of a $S_{\text {expanded }}$ which will eventually characterize the behavior of the DOR module.

\subsection{Performance Results}

To locate two desirable controlling parameters for a given disk, we take the following two steps. First, while strictly maintaining a given QoS feature, we seek a $M_{d m o}^{\text {best }}$ which gives the most proper adjustment to the disk overhead so that it can maximize disk I/O performance. Second, by relaxing the degree of QoS enforcement with the $M_{d m o}^{\text {best }}$, we attempt to obtain an $M_{q o s}^{\text {best }}$ which provides a better disk I/O performance with acceptable QoS deterioration. Finally, the proposed algorithm with $M_{d m o}^{\text {best }}$ and $M_{\text {qos }}^{\text {best }}$ will be compared with the YFQ algorithm in terms of the disk I/O performance and QoS guarantee.

Finding a $M_{d m o}^{\text {best }}$ controlling parameter: Recall that the $M_{d m o}$ was invented to control the slack time for overhead associated with disk head movement between two I/O requests. Given a fixed $M_{q o s}$, a larger $M_{d m o}$ will increase the number of I/O requests squeezed into $S_{\text {base }}$ to exploit the estimated overhead of disk head movements. While no I/O requests are allowed to pass through the DOR module with $M_{d m o}=0$, most I/O requests within $R Q$ bypass the DOR module with $M_{d m o}=100$ whose margin is equal to a disk full seek time. Figure 2 shows the I/O throughput of the proposed algorithm with a different $M_{d m o}$ under various $\mathrm{I} / \mathrm{O}$ workload levels where $M_{q o s}=0.5^{1}$. We expect that the given QoS feature will be preserved throughout all I/O workload levels because of $M_{\text {qos }}=0.5$, which corresponds to a strong QoS enforcement. Based on the performance results in Figure 2, the best I/O throughput can be obtained when $M_{d m o}=20$.

To validate the obtained $M_{d m o}$, we investigate the variation of $\mathrm{I} / \mathrm{O}$ throughputs as a function of $M_{d m o}$ under a fixed I/O workload level, as shown in Figure 3.

\footnotetext{
${ }^{1}$ By definition in Property 2, $M_{q o s}=0.5$ corresponds to a marginal virtual time in the DOR module for either five(5) I/O requests from $R Q_{1}$ or about a single I/O requests from $R Q_{2}$ on the average.
} 
As with the previous results, the maximum I/O throughput can be achieved when $M_{d m o}=20$. Observe that a large $M_{d m o}$ close to 100 cannot improve the I/O throughput due to improper overhead estimations. Conversely, a small $M_{d m o}$ decreases the probability that the remaining I/O requests within $R Q$ can utilize the existing disk overhead time. A length of $S_{\text {expanded }}$ is saturated to about 8

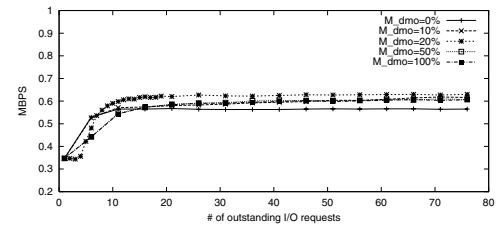

(a) I/O throughput of $R Q_{1}$

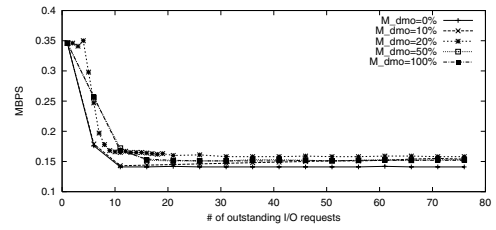

(b) I/O throughput of $R Q_{2}$

Fig. 2. Variations of I/O throughputs of $R Q_{1}$ and $R Q_{2}$ as a function of an I/O workload level by varying the number of outstanding I/O requests $(|I O|)$ under different values of $M_{d m o}$, where $M_{q o s}=0.5$

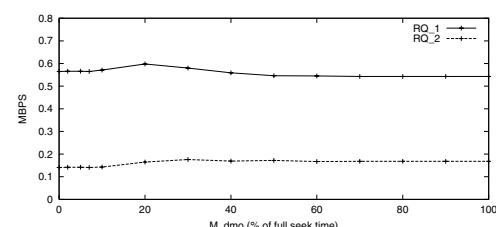

(a) $|I O|=11$

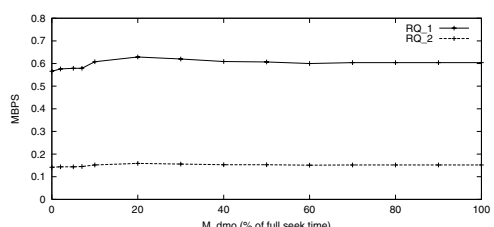

(b) $|I O|=61$

Fig. 3. Variations of $\mathrm{I} / \mathrm{O}$ throughputs as a function of $M_{d m o}$ when $|I O|=11$ and $|I O|=61$, where $M_{\text {qos }}=0.5$

when $M_{d m o}$ becomes 20 . Finally, we conclude the first performance evaluation with the observation that $M_{d m o}^{b e s t}$ of 20 maximizes the I/O throughput under the condition of a strict QoS enforcement.

Finding a $M_{\text {qos }}^{\text {best }}$ controlling parameter: We can achieve an additional performance enhancement by relaxing the degree of QoS enforcement. Figure 4 depicts the variations of I/O throughputs under different $M_{q o s}$ values, where the $M_{d m o}$ is set to 20. The resulting performance of $M_{q o s} \geq 5$ is higher than that of $M_{\text {qos }}=0.5$ by over $10 \%$ in terms of the aggregate $\mathrm{I} / \mathrm{O}$ throughputs of $R Q_{1}$ and $R Q_{2}$. When considering a further improvement in I/O throughputs by increasing $M_{q o s}$, we have to consider the corresponding QoS deterioration. We will examine the variation of $\mathrm{I} / \mathrm{O}$ throughput at each reservation queue as a function of $M_{q o s}$ under fixed I/O workload levels, $|I O|=11$ and $|I O|=61$, as shown 
in Figures 5-6. Figure 5 shows that the I/O throughput of the $R Q_{1}$ decreases, whereas the $\mathrm{I} / \mathrm{O}$ throughput of its competing $\mathrm{I} / \mathrm{O}$ workload $R Q_{2}$ improves by increasing $M_{\text {qos }}$.

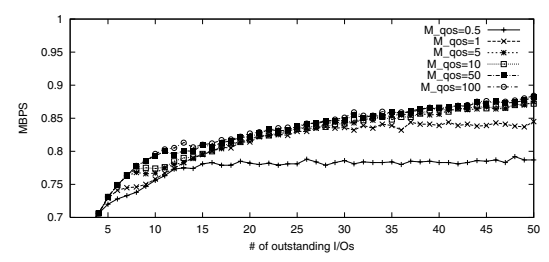

Fig. 4. Variations of I/O throughputs as a function of an I/O workload level under different $M_{\text {qos }}$ values

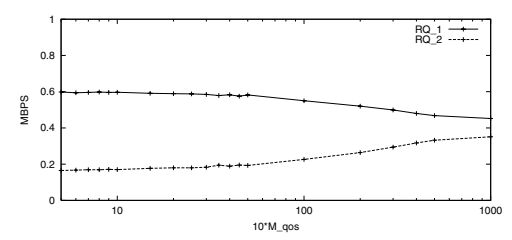

(a) $|I O|=11$

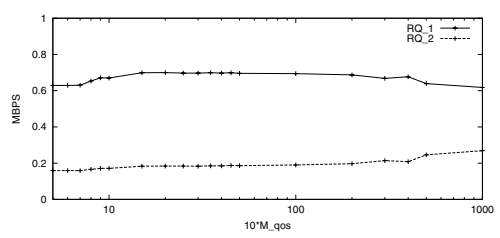

(b) $|I O|=61$

Fig. 5. Variations of I/O throughputs as a function of $M_{\text {qos }}$ under fixed I/O workload levels, $|I O|=11$ and $|I O|=61$, where $M_{d m o}=20$

Figure 6(a)-(b) present the BQS_IO and DOR_IO throughputs which constitute the aggregate I/O throughputs in Figure 5. As $M_{\text {qos }}$ increases with $M_{\text {qos }}=20$, the resulting $\mathrm{I} / \mathrm{O}$ throughput of each reservation queue becomes dominated by the DOR_IO, not the BQS_IO. Note that the BQS_IO consistently preserves the given QoS feature at any level of the I/O workload. However, the resulting I/O throughput of the BQS_IO is diminished as the DOR_IO becomes dominant.

Figure 7 presents that $M_{q o s}$ of 0.5 strongly enforces the given QoS feature to its DOR_IO requests, so that it can provision disk bandwidth with a given ratio of $4: 1$ between $R Q_{1}$ and $R Q_{2}$. However, as $M_{\text {qos }}$ becomes larger, the desirable ratio deteriorates, i.e., the $\mathrm{I} / \mathrm{O}$ throughput of $R Q_{1}$ is mainly affected by $R Q_{2}$, which uses more disk bandwidth with a relaxed QoS enforcement. As a result, the given QoS feature deteriorates by $1 \%$ with $M_{q o s}=0.5$, by $2 \%$ with $M_{q o s}=1$, and by $5 \%$ with $M_{q o s}=5$. It is difficult to precisely determine the metric for the level of QoS satisfaction. In this paper, however, we simply define a ratio of reservation weights as our QoS metric. Using this QoS metric, we have to establish an acceptable range of the QoS satisfaction for a given QoS feature. We believe that satisfying a given QoS feature with $98-100 \%$ accuracy is reasonable 


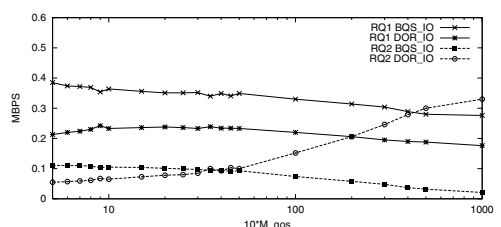

(a) $|I O|=11$

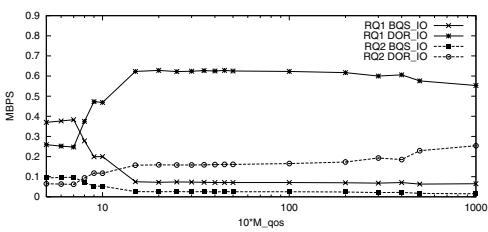

(b) $|I O|=61$

Fig. 6. Variations of BQS_IO and DOR_IO throughputs as a function of $M_{\text {qos }}$ under fixed I/O workloads, $|I O|=11$ and $|I O|=61$, where $M_{d m o}=20$

enough. In that sense, we can say that the QoS margin of $0.5 \leq M_{\text {qos }}^{\text {best }} \leq 1$ falls into such a reasonable range.

Analyzing the degree of QoS guarantee and I/O throughput with the $M_{d m o}^{\text {best }}$ and $M_{q o s}^{\text {best }}$ : We have found $M_{d m o}^{\text {best }}=20$ and $0.5 \leq M_{q o s}^{\text {best }} \leq 1$ maximize the disk I/O performance with negligible QoS deterioration. Hereafter, we compare the proposed algorithm with the baseline algorithm (YFQ) assuring QoS guarantee in terms of the degree of QoS enforcement and I/O throughput. Figure 8(a) shows the degree of QoS enforcement for the given QoS ratio of 80:20 as a function of the number of outstanding I/Os. As expected, the proposed algorithm with $M_{q o s}=0.5$ and $M_{q o s}=1$ maintained the given QoS ratio with more than $98 \%$ accuracy. While the baseline algorithm having $M_{q o s}=0$ provides the given 4:1 ratio properly, the $M_{q o s}=0.5$ and $M_{q o s}=1$ end up with 3.99:1 and 3.96:1, respectively. Figure 8(b) compares the maximum I/O throughput of the proposed algorithm with the $M_{d m o}^{\text {best }}$ and $M_{q o s}^{\text {best }}$ with the case of $M_{q o s}=0$ (YFQ). Observe that the proposed algorithm with $M_{q o s}=0.5$ and $M_{q o s}=1.0$ improves the I/O throughputs by 11-19\% with only 1-2\% QoS deterioration.

\section{Conclusion and Future Work}

We proposed a new proportional-share disk scheduling algorithm that considers both disk characteristics and QoS guarantees in an integrated manner. With extensive simulations, we have discovered two desirable controlling parameters for a given QoS feature. First, given a strong QoS enforcement with $M_{q o s}=0.5$, we observed that the proposed algorithm with $M_{d m o}=20$ achieved the best performance. Second, given the $M_{d m o}=20$, we learned that a better disk I/O performance is achievable with less than $2 \%$ QoS deterioration by increasing $M_{q o s} \leq 1$. Obviously, a serious QoS deterioration is observed as $M_{q o s}$ increases continuously. Finally, comparing the proposed algorithm with the baseline algorithm (YFQ) strictly providing QoS guarantee with $M_{d m o}=0$ and $M_{q o s}=0$, revealed that the proposed algorithm with $M_{d m o}=20 \%$ of the disk full seek time and $0.5 \leq M_{q o s} \leq 1$ improved I/O throughput by $11-19 \%$ with only $1-2 \%$ QoS 


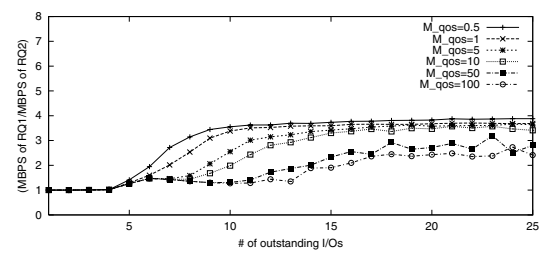

Fig. 7. Variations of QoS guarantees as a function of $M_{q o s}$

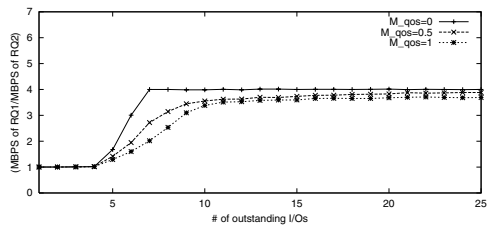

(a) QoS guarantees

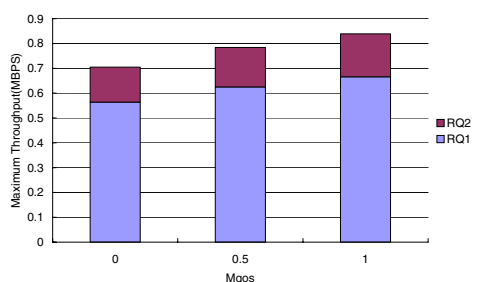

(b) Maximum throughput

Fig. 8. Variation of QoS guarantees and the maximum I/O throughput according to $M_{\text {qos }}$ with $M_{d m o}=20: M_{\text {qos }}=0(\mathrm{YFQ})$ and $M_{\text {qos }}^{\text {best }}=\{0.5,1.0\}$

deterioration. In future work, we need to define a QoS metric which can measure the degree of QoS enforcement and deterioration more accurately. Besides, we need to devise a technique to adjust the two controlling parameters to maximize underlying disk I/O throughputs with tolerable QoS deterioration for any given $\mathrm{I} / \mathrm{O}$ workload including traced real $\mathrm{I} / \mathrm{O}$ workloads.

\section{References}

1. Shenoy, P., Vin, H.: Cello: A disk scheduling framework for next-generation operating systems. In: Proceedings of ACM SIGMETRICS. (1998)

2. Bruno, J., et. al.: Disk scheduling with quality of service guarantees. In: Proceedings of the IEEE International Conference on Multimedia Computing and Systems. (1999)

3. Parekh, A., Gallager, R.: A generalized processor sharing approach to flow control in integrated services networks: The single-node case. IEEE/ACM Trans. on Networking 1 (1993) 344-357

4. Goyal, P., Vin, H., Cheng, H.: Start-time fair queueing: A scheduling algorithm for integrated services packet switching networks. IEEE Transactions on Networking $\mathbf{5}$ (1997) 690-704

5. Schindler, J., Ganger, G.: Automated disk drive characterization. Technical Report CMU-CS-99-176, CMU (1999)

6. Ganger, G., Worthington, B., Patt, Y.: The DiskSim Simulation Environment Version 2.0 Reference Manual. CMU. (1999) 